\section{Endoscopic ultrasound-guided ethanol ablation of pancreatic remnant following complicated total pancreatectomy}
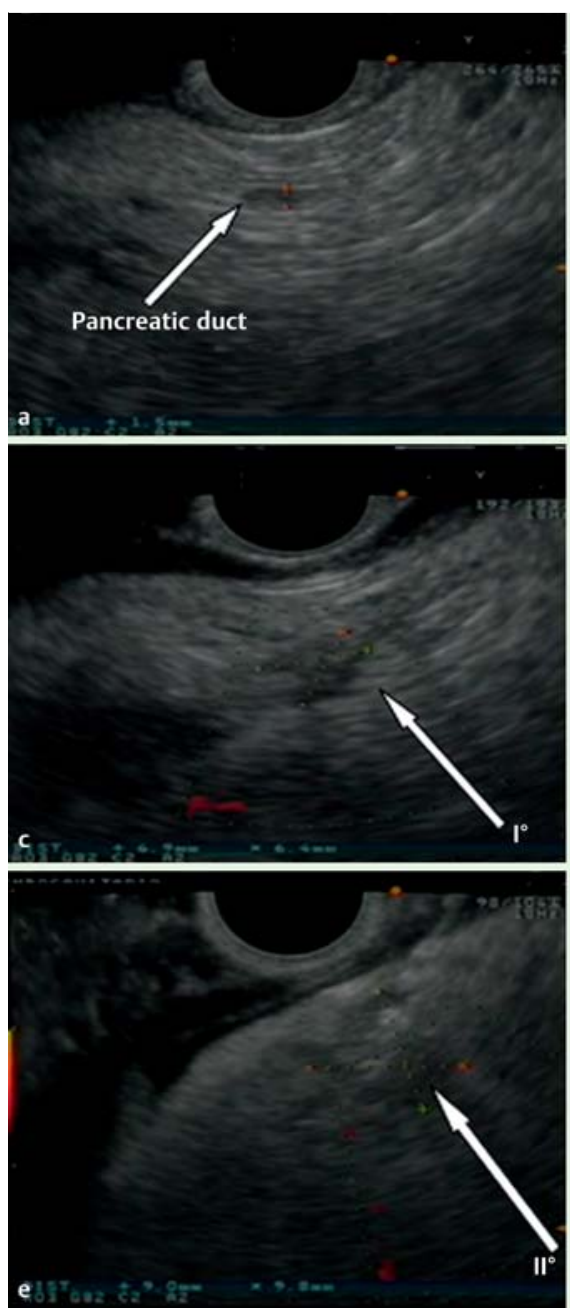

Fig. 1 a Pancreatic remnant with small visible pancreatic duct (arrow) at endoscopic ultrasound in a 59-year-old man who had undergone planned total pancreatectomy but had continuous abdominal drainage with markedly elevated amylase/lipase levels. b Pancreatic remnant (arrow) on the computed tomography scan. c First ethanol injection: hyperechoic area with hypoechoic border visible on endoscopic ultra-

Endoscopic ultrasound (EUS)-guided ethanol ablation of pancreatic tissue has been documented in pigs $[1,2]$ and in humans in pancreatic insulinomas $[3,4]$ and in pancreatic cysts [5]. No data are available on the effect of ethanol injection into normal human pancreas, including accidental injection during treatment of pancreatic lesions.
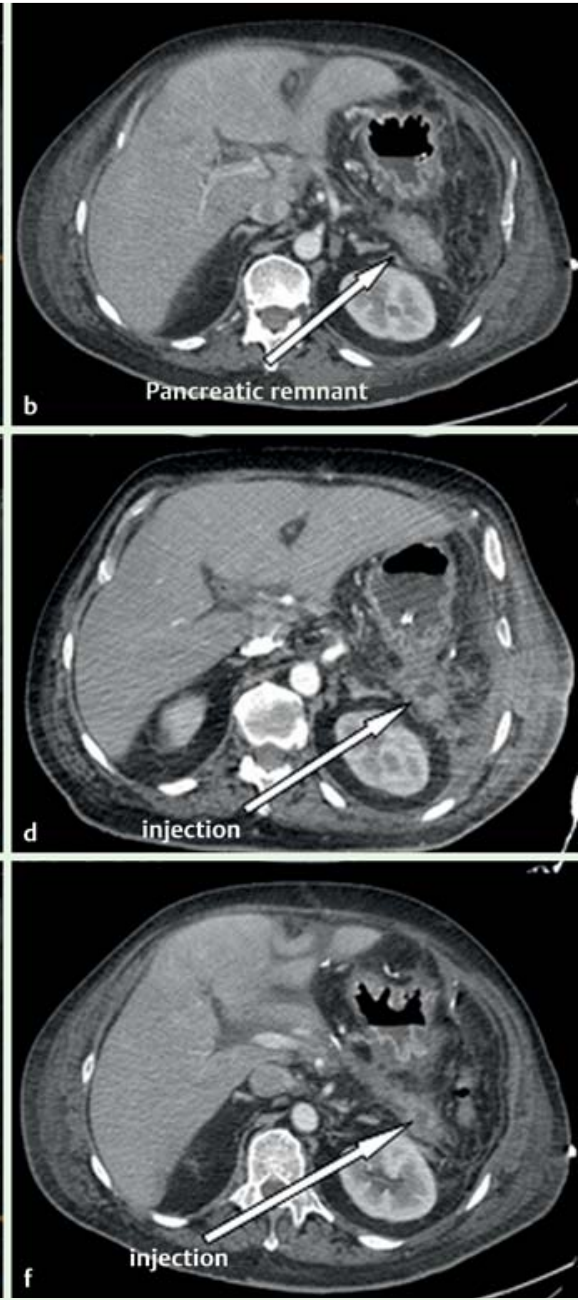

sound soon after injection (arrow). d First ethanol injection: hypodense areas visible on the computed tomography scan 4 days after injection (arrow). e Second ethanol injection: hyperechoic area with hypoechoic border visible on endoscopic ultrasound soon after injection (arrow). f Second ethanol injection: hypodense areas visible on the computed tomography scan 4 days after injection.

A 59-year-old man underwent total pancreatectomy following duodenocephalopancreatectomy for carcinoma of the distal common bile duct, which was complicated by anastomotic dehiscence of the jejunopancreatic anastomosis. Postoperatively he had continuous abdominal drainage of $150-200 \mathrm{~mL} /$ day of fluid with markedly elevated amylase/lipase levels.
An abdominal computed tomography (CT) and EUS showed a pancreatic remnant, $5 \mathrm{~cm}$ in length, with moderate abdominal effusion ( Fig.1a,b). During the next 2 months the patient had recurrent septic episodes, with culture from abdominal effusion positive for polymicrobial infection, and he was admitted into intensive care. Surgery was contraindicated due to the patient's poor condition and selective embolization of the pancreatic stump was not possible due to the inability to identify a vessel in the residual pancreas on the arteriogram. After informed consent, the patient was treated with two EUS-guided ethanol injections in the pancreatic remnant, the first with $1.5 \mathrm{~mL}$ of $96 \%$ alcohol, and the second, 1 week later, with $2.5 \mathrm{~mL}$ of $96 \%$ alcohol, resulting in a $6.9 \times 6.4 \mathrm{~mm}$ and $9.1 \times 7.1 \mathrm{~mm}$ hyperechoic area with a hypoechoic border, respectively, on EUS ( Fig. 1 c,e and Video 1 ). After both injections, the patient experienced opioidresponsive, severe abdominal pain, which lasted for 2 days, but there was no concomitant peak in the serum amylase/lipase levels. A CT scan taken on day 4 after each injection showed ovoid hypodense areas ( $8 \mathrm{~mm}$ and $13.8 \mathrm{~mm}$, respectively) in the pancreatic remnant ( $\mathbf{F i g} \mathbf{1} \mathbf{1}$, f). The abdominal drainage decreased gradually and stopped completely 3 days after the second ethanol injection. The patient's condition progressively improved and he was discharged.

The present report is, to the best of our knowledge, the first documented case of EUS-guided ethanol injection for pancreatic remnant ablation. The observed effect was localized pancreatic damage without elevation of amylase/lipase levels, which is very similar to that seen in experimental pigs treated with alcohol injection [2]. There is a close temporal relationship between the time of administration of the ethanol injection and abdominal effusion healing and patient improvement. This report adds to the knowledge base on the effects of ethanol injection into human pancreas, keeping in mind that we applied the treatment in a pancreatic remnant and not in the intact pancreas.

\section{Video 1}

Endoscopic ultrasound (EUS)-guided ethanol injections into pancreatic remnant. 


\section{Endoscopy_UCTN_Code_TTT_1AS_2AD}

\section{Competing interests: None}

\section{Barresi ${ }^{1}$, A. Granata ${ }^{1}$, I. Tarantino ${ }^{1}$, G. Curcio ${ }^{1}$, N. Azzopardi ${ }^{1}$, C. Ricotta ${ }^{2}$, V. Carollo ${ }^{3}$, G. Marrone ${ }^{3}$, A. Luca ${ }^{2}$, M. Spada², M. Traina ${ }^{1}$}

${ }^{1}$ Gastroenterology and Endoscopy Unit, ISMETT, Palermo, Italy

2 Surgical Department and Liver Transplantation Unit, ISMETT, Palermo, Italy

${ }^{3}$ Radiology Department, ISMETT, Palermo, Italy

\section{References}

1 Aslanian H, Salem RR, Marginean $C$ et al. EUS-guided ethanol injection of normal porcine pancreas: a pilot study. Gastrointest Endosc 2005; 62: 723-727

2 Mattehes K, Mino-Kenudson M, Sahani DV et al. Concentration-dependent ablation of pancreatic tissue by EUS-guided ethanol injection. Gastrointest Endosc 2007; 65: 272 277

3 Jürgensen C, Schuppan D, Neser F et al. EUSguided alcohol ablation of an insulinoma. Gastrointest Endosc 2006; 63: 1059-1062

4 Vleggaar FP, bij de Vaate EA, Valk GD et al. Endoscopic ultrasound-guided ethanol ablation of a symptomatic sporadic insulinoma. Endoscopy 2011; 43: E328 -E329

5 Endoscopic ultrasonography-guided ethanol lavage with paclitaxel injection treats patients with pancreatic cysts. Gastroenterology 2011; 140: 172-179

\section{Bibliography}

DoI http://dx.doi.org/

10.1055/s-0033-1344164

Endoscopy 2013; 45: E195-E196

(c) Georg Thieme Verlag KG

Stuttgart · New York

ISSN 0013-726X

\section{Corresponding author}

\section{Barresi}

Gastroenterology and Endoscopy Unit ISMETT

Via Tricomi 1

Palermo 90100

Italy

Fax: +39-091-2192400

Ibarresi@ismett.edu 